\title{
FREE TOPOLOGICAL GROUPS
}

\author{
B. R. GELBAUM ${ }^{1}$
}

0 . Introduction. In $[3 ; 6 ; 11]$ the basic questions of the existence and of the uniqueness of free topological and free topological abelian groups are discussed. Below we present a different approach to these questions and then indicate the consequences of this approach. Whereas Markov [11] resorts to norms and multinorms, Graev [3] to metrics and Kakutani [6] to topological products of large families of groups, we use very special groups of homomorphisms. Inasmuch as our construction may be viewed as a topological product, retopologized, our ideas are most closely related to those of Kakutani.

1. Free topological groups. If $X$ is a completely regular space [7], the free topological group $F(X)$ is defined as a topological group such that:

(i) $X$ is topologically embeddable in $F(X)$;

(ii) When embedded as in (i), $X$ generates $F(X)$;

(iii) If $\phi$ is a continuous mapping of $X$ into any topological group $G$, there is an extension $\Phi$ of $\phi$ from $X$ to $F(X)$ such that $\Phi$ is a continuous homomorphism of $F(X)$ into $G$.

Let $F_{0}(X)$ be the free group generated by the elements of $X$ serving as indeterminates. Thus $F_{0}(X)$ is the totality of finite words $x_{1}^{\epsilon_{1}} x_{2}^{\epsilon_{2}} \cdots x_{n}^{e_{n}}, \epsilon_{i}= \pm 1, x_{i} \in X$ with the usual convention for equality, triviality and multiplication. If the family $\mathfrak{F}$ of group topologies on $F_{0}(X)$ for which $X$ is naturally embedded topologically is nonempty, then [3] the supremum of $\mathfrak{F}$ is the topology that makes $F_{0}(X)$ into a group $F(X)$ satisfying (i)-(iii). Thus the question of existence turns on the nonemptiness of $\mathfrak{F}$. (The question of uniqueness is settled at once in terms of (iii).)

We proceed as follows: Let $Q^{*}(X)$ be the set of bounded continuous quaternion-valued functions that are never zero on $X$. Let $\mathfrak{Q}$ be the group of elements in $Q^{*}(X)$ that have reciprocals in $Q^{*}(X)$, i.e., $\mathfrak{Q}$ is the set of elements invertible in the multiplicative structure of $Q^{*}(X)$. For each $x$ in $X$ define the homomorphism $x: \mathfrak{Q} \rightarrow Q^{*}$ (the multiplicative group of nonzero quaternions) by the formula $x(f)$ $=f(x)$ for $f \in \mathfrak{Q}$. Let $H=\operatorname{Hom}_{c}\left(\mathfrak{Q}, Q^{*}\right)$ (continuous homomorphisms

Presented to the Society, September 1, 1960; received by the editors July 8, 1960 and, in revised form, October 17, 1960.

1 This research was sponsored in part by the National Science Foundation, Grant No. NSF-G11048. The author is also indebted to B. Jonsson and G. Kalisch for the opportunity to discuss with them the subject of this paper. 
only) and let $H$ be given the weak topology as follows: A typical neighborhood of the identity $e$ in $H$ is a set

$$
U=\left\{h|| h\left(f_{i}\right)-1 \mid<\epsilon, i=1,2, \cdots, n\right\}
$$

where $\epsilon>0, f_{i} \in \mathfrak{Q}$ and $|\cdots|$ denotes the quaternionic norm. It is easily verified that in this topology $H$ is a topological group. Furthermore, since $X$ is completely regular, distinct points of $X$ correspond to distinct members of $H$. Hence we regard $X$ as a subset of $H$ and prove

Theorem 1. $X$ is contained topologically in $H$.

Proof. A typical neighborhood of an $x_{0} \in X$ is a set $U=\left\{h|| h\left(f_{i}\right) x_{0}^{-1}\left(f_{i}\right)-1 \mid<\epsilon, \quad i=1, \quad 2, \cdots, n\right\} . \quad$ But $V=\left\{x|| f_{i}(x)\left(f_{i}\left(x_{0}\right)\right)^{-1}-1 \mid<\epsilon, i=1,2, \cdots, n\right\} \subset U$, whence the embedding is continuous. On the other hand, if $V$ is a neighborhood of $x_{0}$ in $X$ there is an $f \in \mathfrak{O}$ such that $f\left(x_{0}\right)=3, f(X \backslash V)=2,2 \leqq f(X)$ $\leqq 3$. Then $U=\left\{h|| h(f) \cdot x_{0}^{-1}(f)-1 \mid<1 / 4\right\}$ is such that $U \cap X \subset V$. Hence $X$ is contained topologically in $H$.

THEOREM 2. Let $H_{0}$ be the least subgroup of $H$ that contains $X$. Then $F_{0}(X)$ and $H_{0}$ are isomorphic.

To prove Theorem 2, we establish the following lemmas.

LEMMA 1. $Q^{*}$ contains a free subgroup having infinitely many generators.

Proof. If $R$ is a rotation in 3-space, then [1], there is a quaternion $q$ such that for each vector $y \equiv\left(y_{1}, y_{2}, y_{3}\right), R(y)=z \equiv\left(z_{1}, z_{2}, z_{3}\right)$ can be found from the equation $z_{1} i+z_{2} j+z_{3} k=q\left(y_{1} i+y_{2} j+y_{3} k\right) q^{\prime}$ where $q^{\prime}$ is the conjugate of $q$. Since $[4 ; 5 ; 8]$ the group of rotations in 3 -space contains a free group having infinitely many generators $R_{1}, R_{2}, \cdots$, the corresponding quaternions $q_{1}, q_{2}, \ldots$ form a system of generators for a free group in $Q^{*}$.

Lemma 2. Let $p_{1}, p_{2}, \cdots, p_{m}$ be distinct points in $X$. Then there is an $f \in \mathfrak{Q}$ such that $f\left(p_{i}\right)= \pm q_{i}, i=1,2, \cdots, m$ ( $q_{i}$ as in Lemma 1$)$.

Proof. Let $V_{i}$ be a neighborhood of $p_{i}, V_{i} \cap V_{j}=\varnothing, i \neq j$. Choose $f_{i} \in C(X)$ (三set of continuous complex-valued functions on $X$ ) so that $0 \leqq f_{i}(X) \leqq 1, f_{i}\left(p_{i}\right)=1, f_{i}\left(X \backslash V_{i}\right)=0$. Let $W_{i}=\left\{x|| q_{i} f_{i}(x) \mid>1 / 2\right\}$ $\subset V_{i}$, and then let $g_{i} \in C(X), 0 \leqq g_{i}(X) \leqq 1, g_{i}\left(X \backslash W_{i}\right)=0, g_{i}\left(p_{i}\right)=1$. Let $g_{0}=\prod_{i}\left(1-g_{i}\right)$ and let $r_{i}$ be the real part (first component) of $q_{i}, \eta_{i}=\operatorname{sgn} r_{i}$. Setting $f=g_{0}+\sum_{i} \eta_{i} q_{i} f_{i}$, we see that $f\left(p_{i}\right)= \pm q_{i}$. Furthermore, we show $|f|$ is bounded away from $0: \operatorname{In} X \backslash \bigcup_{i} V_{i}, f=1$; 
in any $V_{i}, f=1-g_{i}+\eta_{i} q_{i} f_{i}$; in $W_{i},|f|>1 / 2$; in $V_{i} \backslash W_{i}, 1-g_{i}=1$ and thus $|f| \geqq 1$. Since $f$ is continuous and $|f|$ is bounded, $f \in \mathfrak{Q}$.

We can prove Theorem 2 by showing that only trivial products $x_{1}^{\epsilon_{1}} x_{2}^{\epsilon_{2}} \cdots x_{n}^{\epsilon_{n}}$ can reduce to the identity in $H$. Thus let $p_{1}, p_{2}, \cdots, p_{m}$ be the distinct points among $x_{1}, x_{2}, \cdots, x_{n}, m \leqq n$. Then for the function $f$ of Lemma 2, clearly $\left(x_{1}^{\epsilon_{1}} x_{2}^{\epsilon_{2}} \cdots x_{n}^{\epsilon_{n}}\right)(f) \neq 1$ unless the product $x_{1}^{\epsilon_{1}} x_{2}^{\epsilon_{2}} \cdots x_{n}^{\epsilon_{n}}$ is trivial.

Thus we have shown that $F_{0}(X)$ is isomorphic to the subgroup $H_{0}$. On the other hand $H_{0}$ contains $X$ topologically. Thus the family $F$ is nonempty and the existence of the free topological group is proved. Note that $H_{0}$ is a subgroup of the product $P_{x} Q_{x}^{*}$ where each $Q_{x}^{*}=Q^{*}$ and $x \in X$. However, $H_{0}$ may require retopologization (strengthening) to convert it to $F(X)$.

To construct $A(X)$, the free topological abelian group generated by $X$, we need only replace $Q^{*}$ by $R^{+}$(the multiplicative group of positive real numbers) and imitate the process described above. There is a curious by-play here: $A(X)$ is the abelianization of $F(X)$ [3], while $R^{+}$is the abelianization of $Q^{*}$. We shall discuss this point at greater length below.

\section{Applications.}

TheOREM 3. $X$ is closed in $F(X)$ (or $A(X))$.

Proof. We offer the proof only for $F(X)$. If $T_{0}$ denotes the topology that $H_{0}$ inherits from $H$, then we show in fact that $X$ is closed relative to $T_{0}$, (a fortiori in $F(X)$ ). Let $h_{0} \in H_{0}$ be in the $T_{0}$-closure of $X$. Hence there is a net [7] $y_{\alpha} \rightarrow h_{0}, y_{\alpha} \in X$. If $h_{0} \in X$, and if $h_{0}=\prod_{i=1}^{n} x_{i}^{\epsilon_{i}}$, then there is an open set $U \supset\left\{x_{1}, x_{2}, \cdots, x_{n}\right\}$ and such that ultimately $y_{\alpha} \notin U$. Thus we assume all $y_{\alpha} \notin U$. Choose $f \in \mathfrak{Q}$ so that $f\left(x_{i}\right)=M+1, f\left(y_{\alpha}\right)=M$. Then we see $M=(M+1)^{K}$ where $K=\sum_{i=1}^{n} \epsilon_{i}$. For $K$ given, we can choose $M$ so that $M \neq(M+1)^{K}$ and a contradiction results.

TheOREM 4. $F(X)$ and $A(X)$ are maximally almost-periodic [6; $10 ; 12]$.

Proof. Again we offer the proof only for $F(X)$. Let $e \neq h_{0} \in H_{0}$. If $h_{0}=\prod_{i=1}^{n} x_{i}^{\epsilon_{i}}$, and if $p_{1}, p_{2}, \cdots, p_{m}, m \leqq n$, are the distinct points among the $x_{i}$, let $f$ be as in Lemma 2 , and set $g=f /|f|$. We remark that $\left|q_{i}\right|=1$, and observe that $h_{0}(g) \neq 1$. In view of the condition $|g|=1$, we see that $h \rightarrow h(g)$ is a homomorphism of $H_{0}$ into the multiplicative group of unit quaternions, i.e. [0] into $U(2, C)$, and $h_{0}(g) \neq 1$.

REMARK. Note that we have shown that the two dimensional unitary representations of $F(X)$ form a separating class for $F(X)$. 
THEOREM 5. Let $G_{A}$ be the associated compact group (Bohr compactification) of $A(X)[13]$. Then $\hat{G}_{A}$, the character group of $G_{A}$, is $K=\{f|f \in C(X)| f \mid, \equiv 1\}$ in the discrete topology.

Proof. If $f \in K$, clearly $f$ engenders a character $c_{f}$ in $A(X)$ and $c_{f}$ may be extended to a character of $G_{A}$. If $f_{1} \neq f_{2}, c_{f_{1}} \neq c_{f_{2}}$. On the other hand, if $c \in \hat{G}_{A}, c$ restricted to $A(X)$ provides a (possibly discontinuous) character of $A(X)$. But since $A(X)$ is generally weakened when embedded in $G_{A}, c$ is in fact continuous relative to the original topology of $A(X)$. Clearly $c(x) \in K$. Finally, if $f=c(x)$, then $c_{f}=c$.

Analogously, we can prove Theorem $5^{\prime}$ below. First we introduce some conventions. If $f: X \rightarrow U(n, C)$ then $f$ engenders in a natural way a unitary representation of $F(X)$. Because $F(X)$ is maximally almost-periodic, any unitary representation of $F(X)$ may be extended uniquely to a unitary representation of the associated compact group $G_{F}$. Call $f$ irreducible if the corresponding representation is irreducible, and call $f_{1}, f_{2}$ equivalent if the corresponding representations are equivalent.

THEOREM $5^{\prime}$. Let $f_{\alpha}$ be a collection of functions $f_{\alpha}: X \rightarrow U\left(n_{\alpha}, C\right)$ such that (i) all $f_{\alpha}$ are irreducible; (ii) no two $f_{\alpha}$ are equivalent; (iii) any irreducible $f$ is equivalent to one (and hence only one) $f_{\alpha}$. Then any irreducible representation of $G_{F}$ arises from an $f$ equivalent to some $f_{\alpha}$.

3. Almost-periodic topology. We know that $F(X)$ has as its topology the supremum of $\mathfrak{F}$. It is of interest to investigate the infimum of $\mathfrak{F}$. To do this we introduce a special family of functions on $F_{0}(X)$. For each $f \in \mathfrak{Q}$, let $\tilde{f}$ denote the extension of $f$ to $F_{0}(X)$ by the formula:

$$
\tilde{f}\left(\prod_{i=1}^{n} x_{i}^{\epsilon_{i}}\right)=\prod_{i=1}^{n}\left(x_{i}(f)\right)^{\epsilon_{i}} .
$$

An elementary argument shows that $\tilde{f}$ is almost-periodic on $F_{0}(X)$ if and only if $|f| \equiv 1$. Let $T_{A P}$ denote the topology on $F_{0}(X)$ imposed by the functions $\tilde{f}$, i.e., $T_{A P}$ is the weakest topology with respect to which all $\tilde{f}$ are continuous.

THEOREM 6. $T_{A P}$ is a group topology for $F_{0}(X)$. In this topology, $X$ is topologicaly embedded as a closed subset of $F_{0}(X) . T_{A P}$ is the infimum of $\mathfrak{F}$.

Proof. The first statement may be verified by explicit calculation. For the second statement we first record the following facts and definitions. 
For any quaternion $q$, let $e^{q}=\sum_{n=0}^{\infty} q^{n} / n !$. Then: (a) $e^{q} \neq 0$; (b) if $r$ is a quaternion and $|r-1|<1$, there is a quaternion $s$ such that $e^{s}=r$; (c) for any quaternion $r,\left\{s \mid e^{s}=r\right\}$ is either empty or a discrete subset of $Q$, the set of all quaternions; (d) if $e^{s}=r,|r|=1$ and if $a$ is real then $\left|e^{a_{s}}\right|=1$.

The infinite free set $\left\{q_{i}\right\}$ in $Q^{*}$ may be chosen so that $\left|q_{i}\right|=1$. If $q$ is a cluster point of $\left\{q_{i}\right\}$, and if $1>\epsilon>0$ is given, let $\left\{r_{j}\right\}$ be a subset of $\left\{q_{i}\right\}$ such that $\left|r_{j}-q\right|<\epsilon / 4$. Then $\left|r_{1}^{-1} r_{j}-1\right|<\epsilon$, and the set $\left\{r_{1}^{-1} r_{j}\right\}$ is free. Let $e^{s_{j}}=r_{1}^{-1} r_{j}=t_{j}$. Note that $s_{j} \neq 0,\left|t_{j}\right|=1$.

Since $T_{A P}$ represents a weakening of $T_{0}$, the embedding of $X$ in $F_{0}(X)$ is continuous relative to $T_{A P}$. On the other hand, if $W$ is open in $X$, and if $x_{0} \in W$, let $f \in C(X)$ be real-valued and such that $f\left(x_{0}\right)=1$, $f(X \backslash W)=0$. Then if $g=e^{i f}$ we see $|g|=1$, whence $\tilde{g}$ is $A P$. Let $V=\left\{h|| g(h)-1|<\epsilon=| e^{i}-1 \mid\right\}$. Then $x_{0} V \cap X \subset W$. For, if $x \in x_{0} V$, then $\left|g\left(x_{0}^{-1} x\right)-1\right|<\left|e^{i}-1\right|$, whence $\left|e^{i f(x)}-e^{i}\right|<\left|e^{i}-1\right|$. Thus $e^{i f(x)}$ $\neq 1, f(x) \neq 0$ and hence $x \in W$. Thus the embedding of $X$ in $F_{0}(X)$ is open relative to $T_{A P}$ and hence $X$ is topologically embedded in $F_{0}(X)$ relative to $T_{A P}$.

We show next that $X$ is closed relative to $T_{A P}$. Let $h_{0}=\prod_{i=1}^{n} x_{i}^{\varepsilon_{i}}$ $\notin X$, and let $p_{1}, p_{2}, \cdots, p_{m}$ be the distinct points among $x_{1}, x_{2}, \cdots$, $x_{n}$. In terms of the preliminary comments we made, choose $\epsilon>0$ so that when $t_{1}, t_{2}, \cdots, t_{m}$ replace $p_{1}, p_{2}, \cdots, p_{m}$ the resulting quaternion $u$ satisfies: $|u-1|<1$. Let $e^{v}=u$. Since $\left\{t_{j}\right\}$ is a free set, $u \neq 1$, $t_{1}, t_{2}, \cdots, t_{m}$. Let $E=\left\{w \mid e^{w}=u\right\}$. Since $|u|=1, u \neq 1$, we see $0,1 \notin E$. Let $L$ be a curve given by $\phi(\lambda), 0 \leqq \lambda \leqq 1$ and such that $L$ avoids $E_{1}=\bigcup_{j=1}^{m} s_{j}^{-1} E$, where $e^{s_{j}}=t_{j}, \phi(0)=0, \phi(1)=1$, and $d\left(L, E_{1}\right)$ $=\Delta>0$. Choose neighborhoods $V_{j}$ of $p_{j}$ such that $V_{j_{1}} \cap V_{j_{2}}=\varnothing$, $j_{1} \neq j_{2}, j=1,2, \cdots, m$, and choose $f_{j} \in C(X)$ so that $f_{j}\left(p_{j}\right)=1$, $f_{j}\left(X \backslash V_{j}\right)=0,0 \leqq f_{j}(X) \leqq 1$. Let $\psi_{j}(x)=s_{j} \phi\left(f_{j}(x)\right), \psi(x)=\sum_{j=1}^{m} \psi_{j}(x)$, $\gamma(x)=\exp [\psi(x)] /|\exp [\psi(x)]|$. Thus $\tilde{\gamma}$ is $A P$. Then $\tilde{\gamma}\left(h_{0}\right)=u$ and there is a $\delta>0$ such that $|\tilde{\gamma}(x)-u| \geqq \delta$ for all $x \in X$, as the following lines show: $\tilde{\gamma}\left(h_{0}\right)=\prod_{i=1}^{n} \gamma\left(x_{i}\right)^{\epsilon_{i}} ; \gamma\left(x_{i}\right)=\exp \left[\psi\left(x_{i}\right)\right] /\left|\exp \left[\psi\left(x_{i}\right)\right]\right|$; $\psi\left(x_{i}\right)=\psi_{j_{0}}\left(p_{j_{0}}\right)=s_{j_{0}}\left(\right.$ where $\left.x_{i}=p_{j_{0}}\right) ; \exp \left[\psi\left(x_{i}\right)\right]=\exp \left[s_{j_{0}}\right]=t_{j_{0}}$, and $\left|\exp \left[\psi\left(x_{i}\right)\right]\right|=\left|t_{j_{0}}\right|=1$. On the other hand, if $x \in X$, then either (a) $x \in V_{j_{0}}$, some $j_{0}$ or (b) $x \in\left(X \backslash \bigcup_{j=1}^{m} V_{j}\right)$. In case (a) $\tilde{\gamma}(x)$ $=\exp \left[\psi_{j_{0}}(x)\right] /\left|\exp \left[\psi_{j_{0}}(x)\right]\right|=\exp \left[s_{j_{0}} \phi\left(f_{j_{0}}(x)\right)\right] / \exp \left[s_{j_{0}} \phi\left(f_{j_{0}}(x)\right] \mid\right.$. Hence $\left.\left|\exp \left[s_{j_{0}} \phi\left(f_{j_{0}}(x)\right)\right]\right|=1, \gamma(x)=\exp \left[s_{j_{0}} \phi f_{j_{0}}(x)\right)\right]$. The exponent is bounded away from $E_{1}$ for all $j_{0}$ and all $x$, whence, for some $\bar{\delta}>0$, $|\gamma(x)-u| \geqq \bar{\delta}$ in this case. In case (b) $f_{j}(x)=0, \psi(x)=0, \gamma(x)=1$ and $\min (\bar{\delta},|1-u|)$ is the required $\delta$. Hence $X$ is closed in $F_{0}(X)$ relative to $T_{A P \text {. }}$

Finally, any group topology on $F_{0}(X)$ relative to which $X$ is em- 
bedded topologically is one for which all functions $\tilde{f}$ are continuous. Thus $T_{A P}$ is the infimum of $\mathfrak{F}$.

4. Identities. At the end of $\$ 1$ we mentioned an interesting relationship between $F(X)$ and $A(X)$ on the one hand and $Q^{*}$ and $R^{+}$ on the other. More explicitly, the situation may be described as follows: Consider the word $w=a b a^{-1} b^{-1}$. In $Q^{*}$, let $N(w)$ be the subgroup generated by all quaternions arising from the substitution in $w$ of quaternions for $a$ and $b$. Then $N(w)$ is closed and normal and $Q^{*} / N(w)=R^{+}$. In $F(X)$ let $\mathfrak{N}(w)$ be the analog of $N(w)$. Then $\mathfrak{N}(w)$ is closed and normal [3] and $A(X)=F(X) / \mathfrak{N}(w)$. Furthermore, $A(X)$ arises by the constructions used when we consider $R^{+}$-valued rather than $Q^{*}$-valued functions on $X$. A natural surmise might be framed as follows: Let $\left\{W_{\lambda}\right\}_{\lambda \in \Lambda}$ be a family of words. Let $N$ be the closed normal subgroup generated in $Q^{*}$ by substitution of quaternions for the variables in these words. Let $S=Q^{*} / N$. Let $\mathfrak{N}$ in $F(X)$ be the analog of $N$ in $Q^{*}$. Then, if $B(X)=F(X) / \mathfrak{R}, B(X)$ arises by the constructions indicated when $S$-valued functions on $X$ are employed. It is our purpose to show this conjecture is false.

Let $\left\{W_{\lambda}\right\}$ consist of one word, $a^{2}$. It is a simple matter to verify that in this case $N=Q^{*}$, since every quaternion $q$ is a square. Hence $S=1$. On the other hand, $\mathfrak{N}_{\neq}^{\subsetneq} F(X)$ as the following lines show: Let $f$ in $\mathfrak{O}$ be identically 2 . Then for arbitrary $x_{0} \in X$ (regarded as a subset of $F(X))$, the neighborhood $U$ of $x_{0}$ given by $\left\{h|| h(f) x_{0}^{-1}(f)-1 \mid<1 / 4\right\}$ does not meet $\mathfrak{N}$. Thus $\mathfrak{N}$ is a proper closed subgroup in $F(X)$ and $B=F(X) / \mathfrak{N}$ is nontrivial. But if $S$ valued functions are used in one construction, then, since $S$ consists of a single number 1 , the resulting construction will be the trivial group, which $B$ is not.

5. Supplement. The author would like to thank the referee for suggesting the following generalizations of the procedures used in this paper.

Replace $Q^{*}$ by a compact connected Lie group $L$ containing a free subgroup with two generators. Hence $L$ contains a free subgroup with infinitely many generators. Use the invariant metric in $L$ (rather than the quaternionic norm in $Q^{*}$ ) to define neighborhoods as in Theorem 1. The "separating" function $f$ of Theorem 1 is replaced by a continuous function $f$ from $X$ to $L$ such that $f\left(x_{0}\right)=1, f(X \backslash V)$ $=a \neq 1$, and $f(X) \subset$ the geodesic arc $(1 a)^{n}$ (a segment of a oneparameter group). Specialize $L$ to $S O(3, R)$ or $U(2, C)$ to get the results given.

Similar techniques may be applied to generalizations of Theorem 6 . 
The following problem now arises: Let $L$ be the Lie-Moufang loop of all Cayley numbers of norm 1. If $L$ can be shown to contain a free Moufang subloop of infinite rank, the procedures described lead to the construction of a free topological Moufang loop containing $X$.

\section{BIBLIOGRAPHY}

0. C. Chevalley, Theory of Lie groups, Princeton, 1946.

1. L. E. Dickson, Modern algebraic theories, New York, 1930.

2. I. Gelfand, Über verschiedene Methoden der Einführung der Topologie in der Menge der maximalen Ideale eines normierten Ringes, Mat. Sb. (N. S.) vol. 9 (1941) pp. 25-39.

3. M. I. Graev, Svobodnie topologičeskie gruppĭ, Izv. Akad. Nauk SSSR. Ser. Mat. vol. 12 (1948) pp. 279-324; Amer. Math. Soc. Translations Series 1, no. 35, 1951.

4. J. De Groot and T. Dekker, Free subgroups of the orthogonal group, Compositio Math. vol. 12 (1954) pp. 134-136.

5. F. Hausdorff, Grundzüge der Mengenlehre, Leipzig, 1914.

6. S. Kakutani, Free topological groups and infinite direct product topological groups, Proc. Imp. Acad. Japan vol. 20 (1944) pp. 595-598.

7. J. L. Kelley, General topology, New York, 1955.

8. A. Kurosh, The theory of groups, 2d edition, New York, 1955.

9. L. Loomis, An introduction to abstract harmonic analysis, New York, 1953.

10. W. Maak, Fastperiodische Funktionen, Grundlehren der mathematischen Wissenschaften, Bd. LXI, Berlin-Göttingen-Heidelberg, Springer-Verlag, 1950.

11. A. A. Markov, O svobodnich topologičeskich gruppach, Izv. Akad. Nauk SSSR. Ser. Mat. vol. 9 (1945) pp. 3-64; Amer. Math. Soc. Translations Series 1, no. 30, 1950, pp. 11-88.

12. T. Nakayama, Note on free topological groups, Proc. Imp. Acad. Japan vol. 19 (1943) pp. 471-475.

13. A. Weil, L'intégration dans les groupes topologiques et ses applications, Paris, 1938.

UNIVERSITY OF MinNESOTA 